\title{
NUTRIENTES EN EL GOLFO DE CALIFORNIA
}

\author{
por \\ SAUL ALVAREZ BORREGO 1 \\ JORGE ALBERTO RIVERA 2 \\ GILBERTO GAXIOLA CASTRO ${ }^{1}$ \\ MANUEL DE JESUS ACOSTA RUIZ ${ }^{1}$. \\ RICHARD A. SCHWARTZLOSE ${ }^{3}$ \\ 1 Centro de Investigación Científica y de \\ Educación Superior de Ensenada, B. Cfa. \\ Espinoza \#843 Ensenada, B. C. \\ México \\ $2 \quad$ Unidad de Ciencias Marinas \\ Universidad Autónoma de Baja California \\ A. P. \#453 Ensenada, B. C. \\ México \\ 3 Scripps Institution of Oceanography \\ University of California at San Diego \\ La Jolla, California, 92037 \\ U. S. A.
}

RESUMEN.

La distribución vertical de nutrientes en el Golfo de California al sur de las islas Angel de la Guarda y Tiburón, es muy similar en general a la del Océano Pacífico Norte Tropical Oriental. Los gradientes en $10 \mathrm{~s} 100 \mathrm{~m}$ superficiales son muy intensos, con valores de más de $2 \mu \mathrm{M}$ de fosfato, de $15 \mu \mathrm{M}$ de nitrato $\mathrm{y} 30 \mu \mathrm{M}$ de silicato a $100 \mathrm{~m}$. En el canal de Ballenas (o cuenca Salsipuedes) la distribución vertical de nutrientes es única, de acuerdo con la distribución de oxígeno, temperatura y salinidad. En el canal de Ballenas los nutrientes aumentan 
monotonicamente con la profundidad hasta $3.0 \mu \mathrm{M}$ de fosfato, $30 \mu \mathrm{M}$ de nitrato y $70 \mu \mathrm{M}$ de silicato, a $1500 \mathrm{~m}$. A $1000 \mathrm{~m}$, el canal de Ballenas tiene $0.6 \mu \mathrm{M}, 12 \mu \mathrm{M}$ y $60 \mu \mathrm{M}$ menos de fosfato, nitrato y silicato respectivamente, que la parte sur del Golfo. La distribución vertical del oxígeno, temperatura, salinidad y nutrientes indican que en el canal de Ballenas había una mayor proporción del agua de la profundidad del umbral en octubre que en abril. Las concentraciones superficiales de nutrientes, de la boca del Golfo hacia el interior, tiene valores máximos en el canal de Ballenas, para disminuir luego en la parte norte. En octubre no se detectó el efecto de surgencias y en abril el muestreo no permitió estudiar este efecto. El nitrito presentó un máximo subsuperficial a 30-80 m en la mayoría de las estaciones, con valores en general entre 0.2 y $0.6 \mu \mathrm{M}, \mathrm{y}$ hasta más de $1 \mu \mathrm{M}$ en el canal de Ballcnas. En la boca del Golfo se presentó un segundo máximo de nitrito a 150-400 m, característico de zonas con bajas concentraciones de oxígeno. No es aparente por qué no se presentó en el interior del Golfo. En el centro del norte del Golfo, en abril se detectaron máximos débiles de fosfato, nitrato y silicato a 80-125 m. Esto y la inversión del gradiente vertical de salinidad abajo de $100 \mathrm{~m}$ en abril, parecen indicar hundimientos de agua superficial en invierno en la cabeza del Golfo, con posterior movimiento hacia el sur.

\section{ABSTRACT.}

In the Gulf of California, south from Angel de la Guarda and Tiburon islands, the vertical distribution of nutrients is very similar in general to that of the Eastern Tropical North Pacific Ocean. From 0 to $100 \mathrm{~m}$, gradients are very intense, with values of more than $2 \mu \mathrm{M}$ for phosphate, $15 \mu \mathrm{M}$ for nitrate and $30 \mu \mathrm{M}$ for silicate at $100 \mathrm{~m}$. In Ballenas channel (or Salsipuedes basin) the vertical distribution of nutrients is unique, in agreement with that of oxygen, temperature and salinity. In this basin, nutrients increase monotonicaly with depth up to $3 \mu \mathrm{M}$ phosphate, $30 \mu \mathrm{M}$ nitrate and $70 \mu \mathrm{M}$ silicate, at $1500 \mathrm{~m}$. At $1000 \mathrm{~m}$, in Ballenas channel, phosphate, nitrate and silicate are $0.6 \mu \mathrm{M}, 12 \mu \mathrm{M}$ and $60 \mu \mathrm{M}$ respectively, less than at the southern 
part of the Gulf. Vertical distributions of temperature, salinity, oxygen and nutrients indicate that in Ballenas channel there was a greater proportion of water from sill depth in october than in april. Nutrient surface concentrations between the mouth of the Gulf and the northern end are the highest over the Ballenas channel. We did not detect the effect of upwelling in october. In april, sampling did not allow to study the effect of upwelling. Nitrite had a subsurface maximum at $30-80 \mathrm{~m}$, at most of the stations, with values in general between 0.2 and $0.6 \mu \mathrm{M}$, and more than $1 \mu \mathrm{M}$ at Ballenas channel. At the mouth of the Gulf, there was a secondary nitrite maximum at $150-400 \mathrm{~m}$. This secondary maximum is characteristic of regions with very low oxygen concentrations. It is not apparent why it did not appear in the interior of the Gulf. In the central northern Gulf, weak phosphate, nitrate and silicate maxima were detected at 80$125 \mathrm{~m}$ in april. These maxima and the inversion of the vertical salinity gradient below $₫ 100 \mathrm{~m}$, shown in april, indicate the posibility of downwelling in winter, at the head of the Gulf, with subsequent southward advection.

\section{INTRODUCCION.}

Desde los tiempos de los primeros exploradores en el siglo XVI, se ha venido mencionando que el Golfo de California tiene una gran fertilidad. Sin embargo, estamos lejos de conocer con detalle la variabilidad de la concentración de nutrientes en el Golfo, en el espacio y en el tiempo. Zeitzschel (1969) revisó los pocos datos disponibles e hizo las siguientes conclusiones: durante verano e invierno, las concentraciones superficiales de fosfato son mayores a 0.4 $\mu \mathrm{M}$ en todo el Golfo, aún en la parte sur; en la parte norte del Golfo, las concentraciones superficiales de fosfato fueron entre $0.9 \mathrm{y} .1 .9 \mathrm{\mu M}$; los datos sugieren que las concentraciones de fosfato en el Golfo están muy por encima del límite establecido experimentalmente de $0.22 \mu \mathrm{M}$ para el crecimiento de diatomeas oceánicas tropicales (Thomas y Dodson, 1968). Warsh, Warsh y Staley (1973) presentaron la distribución vertical de fosfato y silicato a través de la boca del Golfo, para julto de 1967. Sus gráficas muestran valores superficiales de fosfato de alrededor de $0.2 \mathrm{mM}$, aumentando rápidamente con la profundidad hasta cerca de 2.3 WM a $100 \mathrm{~m}, \mathrm{y}$ a un máximo de alrededor de 3.4 $\mathrm{m}$ a $800 \mathrm{~m}$. 
Los valores superficiales de siliaato fueron cerca de $2,1 M$, aumentando monotónicamente con la profundidad hasta alrededor de $100 \mathrm{MM}$ a 800-1000 m. En los $50 \mathrm{~m}$ superficiales, ambos fosfato $y$ silicato fueron mayores cerca de la costa oeste, probablemente debido a la surgencia durante verano en esta costa.

El B/I "Alexander Agassiz" de la Institución Scripps de Oceanografía, colectó datos de fosfato, nitrato, nitrito y silicato en dos cruceros al Golfo de California en 1974. Fn este trabajo se reportan dichos datos, describiendo las condiciones de primavera y otoño para todo el Golfo.

\section{METODOS.}

Los muestreos se realizaron del 8 de abril al 4 de mayo $y$ del 5 al 31 de octubre de 1974. De las estaciones muestreadas en el primer crucero se escogieron 15 para construir secciones de distribución vertical de los nutrientes, en el sentido longitudinal del Golfo, de la boca al extremo interno, pasando por el canal de Ballenas o cuenca Salsipuedes (Fig. la); y se escogieron 14 del segundo crucero para el mismo fin (Fig. Ib). En el segundo crucero se ocuparon estaciones hidrográficas de tal manera de también permitir el construir secciones de distribución vertical, transversal al Golfo, para el norte de la cuenca de Guaymas y el norte de la cuenca Delfín, así como de la cuenca Tiburón. Pero estas últimas secciones no muestran información adicional a las longitudinales, las isogramas son en general muy horizontales, así que no se presentan en este trabajo, sólo se hace mención en el texto de algunas características de interés. La localización geográfica de las estaciones del primer crucero no permite el estudiar los efectos de surgencia, porque no están alineadas perpendicular a la costa.

Para la obtención de las muestras se utilizaron botellas Nansen recubiertas con teflón, con termómetros reversibles. Para la determinación de oxígeno se utilizó el método microWinkler. La determinación de los nutrientes se hizo de acuerdo con el Manual del Técnico Marino de Scripps (STO, 1974), utilizando un espectrofotómetro Beckman DU. Todos los análisis se hicieron a bordo. La salinidad se determinó con un salinómetro de inducción Bissett-Berman, modelo 6230 .

Para estimar la precisión de las determinaciones de nutrientes se tomaron datos de las estaciones más profundas de los cruceros, en la boca y fuera del Golfo al oeste de cabo San Lucas. Entre 2200 y $2700 \mathrm{~m}$, aproximadamente, hay 2 ó 3 datos por estación que varían irregularmente con la profundidad, 

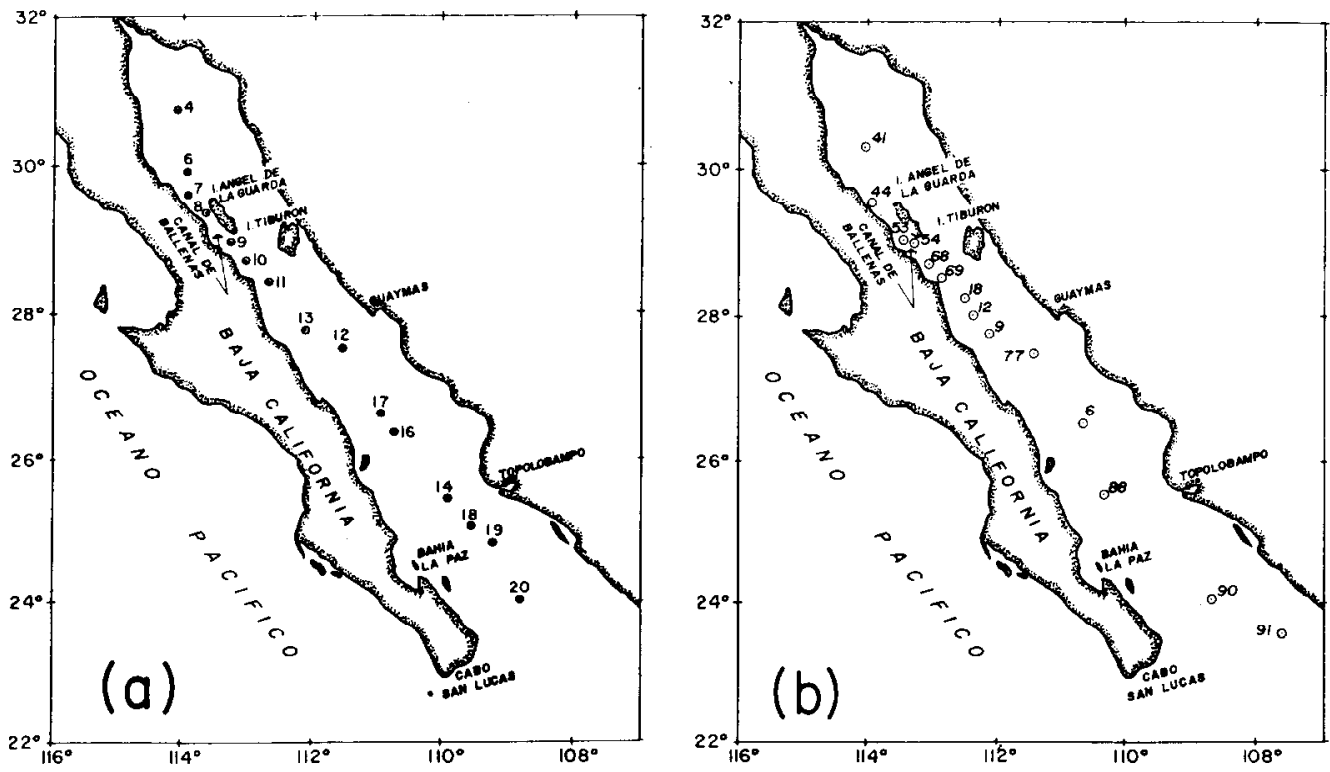

Fig. 1. Localización de las estaciones hidrográficas. a) crucero de abril-mayo, 1974; b) crucero de octubre, 1974.

es decir, sus valores giran alrededor de una media sin un patrón particular ( $\mathrm{g} . \mathrm{g}_{\text {. : }}$ no disminuyen monotónicamente con la profundidad). Desafortunadamente hubo muy pocas estaciones que alcanzaron estas profundidades de muestreo. Sin embargo, tomando en total siete valores para cada nutriente de tres estaciones del primer crucero, y diez valores para cada nutriente de tres estaciones del segundo crucero, obtuvimos las siguientes estimaciones para una desviación estandar, de fosfato, nitrato y silicato, respectivamente: primer crucero $1 \%, 2 \%$ y $3.3 \%$; segundo crucero $4 \%, 5 \%$ y $2 \%$; ambos cruceros juntos $3 \%, 3.7 \%$ y $2.5 \%$.

RESULTADOS,

La distribución vertical de temperatura, salinidad y oxígeno disuelto mostró características que han sido reportadas en general por otros autores (Sverdrup, 1941; Roden, 1964) (Figs. 2 y 3). Gaxiola Castro, Alvarez Borrego y Schwartzlose (1978) presentaron y discutieron la distribución vertical que resultó del crucero de abril-mayo (Figs. 2a, c y 3a). Aquí haremos hincapié únicamente en las diferencias entre octubre y abrilmayo. El umbral sur de la cuenca Salsipuedes, entre isla Angel de la Guarda y la península, marca la separación entre dos 

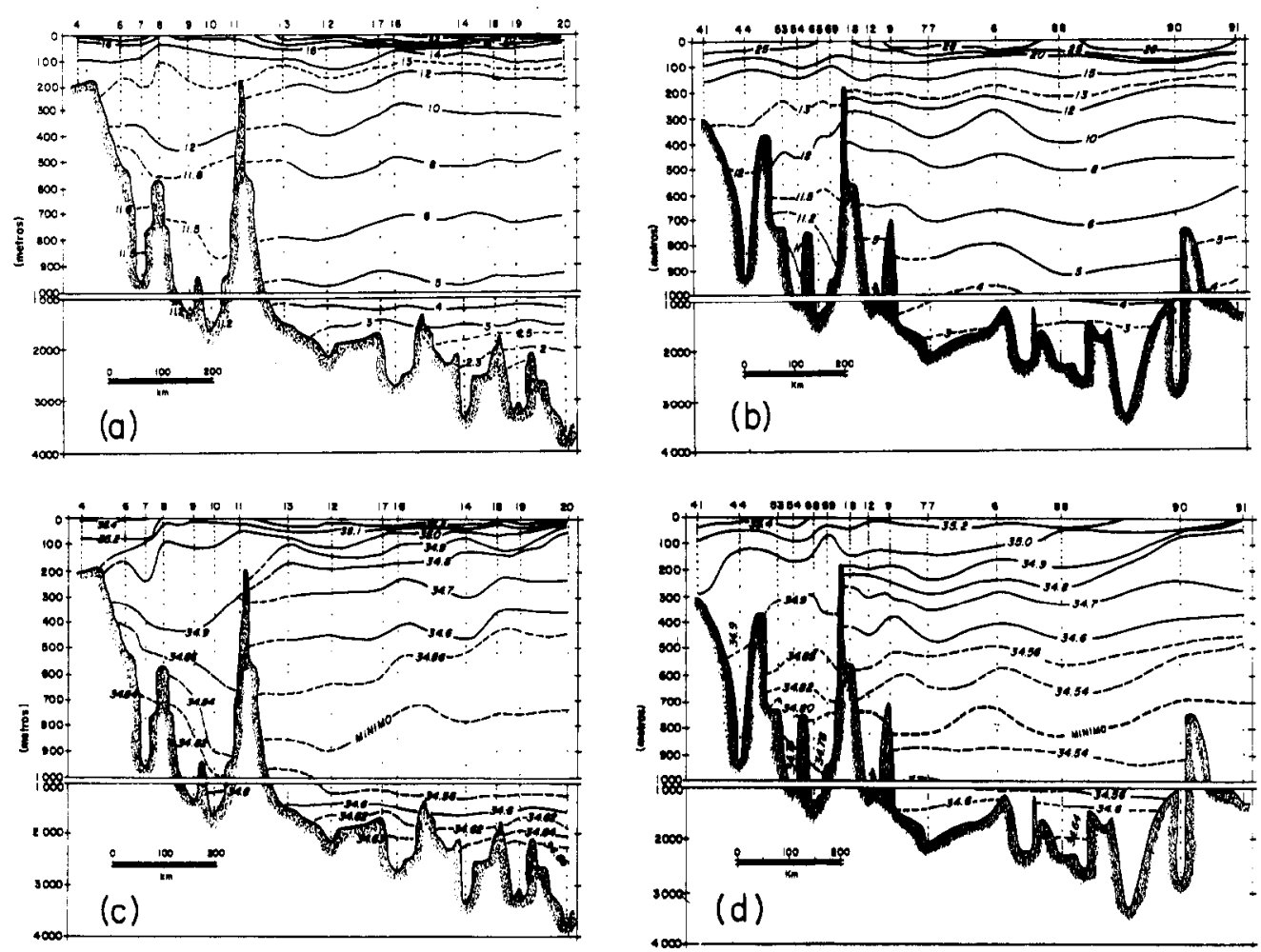

Fig. 2. Distribución vertical: a) temperatura en abril-mayo $\left({ }^{\circ} \mathrm{C}\right)$; b) temperatura en octubre $\left({ }^{\circ} \mathrm{C}\right)$; c) salinidad en abril-mayo $\left(\% /{ }^{\circ}\right) ; y$ d) salinidad en octubre $(\% \circ)$. a y c son tomadas de Gaxiola Castro, Alvarez Borrego y Schwartzlose (1978). En la parte superior se marcan los números de las estaciones.

zonas hidrográficas diferentes. Ia parte del Golfo al sur de este umbral, que denominaremos gubsecuentemente parte sur, tiene una distribución espacial de las propledades del agua similar a la del Pacfifico Orlental Tropical en general. Mientras que la parte al norte de este umbral tiene distribuciones verticales distintas $\mathrm{y}$ únicas. Las propiedades del agua profunda de cuenca Salsipuedes o canal de Ballenas (estaciones 53,54,68 y 69, Fig. Ib) tienden a tener los mismos valores que las de la profundidad del umbral. La profundidad máxima del umbral sur de cuenca Salsipuedes es alrededor de $450 \mathrm{~m}$ (Rusnak, Fisher y Shepard, 1964), aunque las gráficas aquf presentadas no la muestran. De acuerdo con Roden (1964), la homogeneización en el canal de Ballenas se debe a los fuertes procesos de mezcla provocados principalmente por corrientes de marea. 

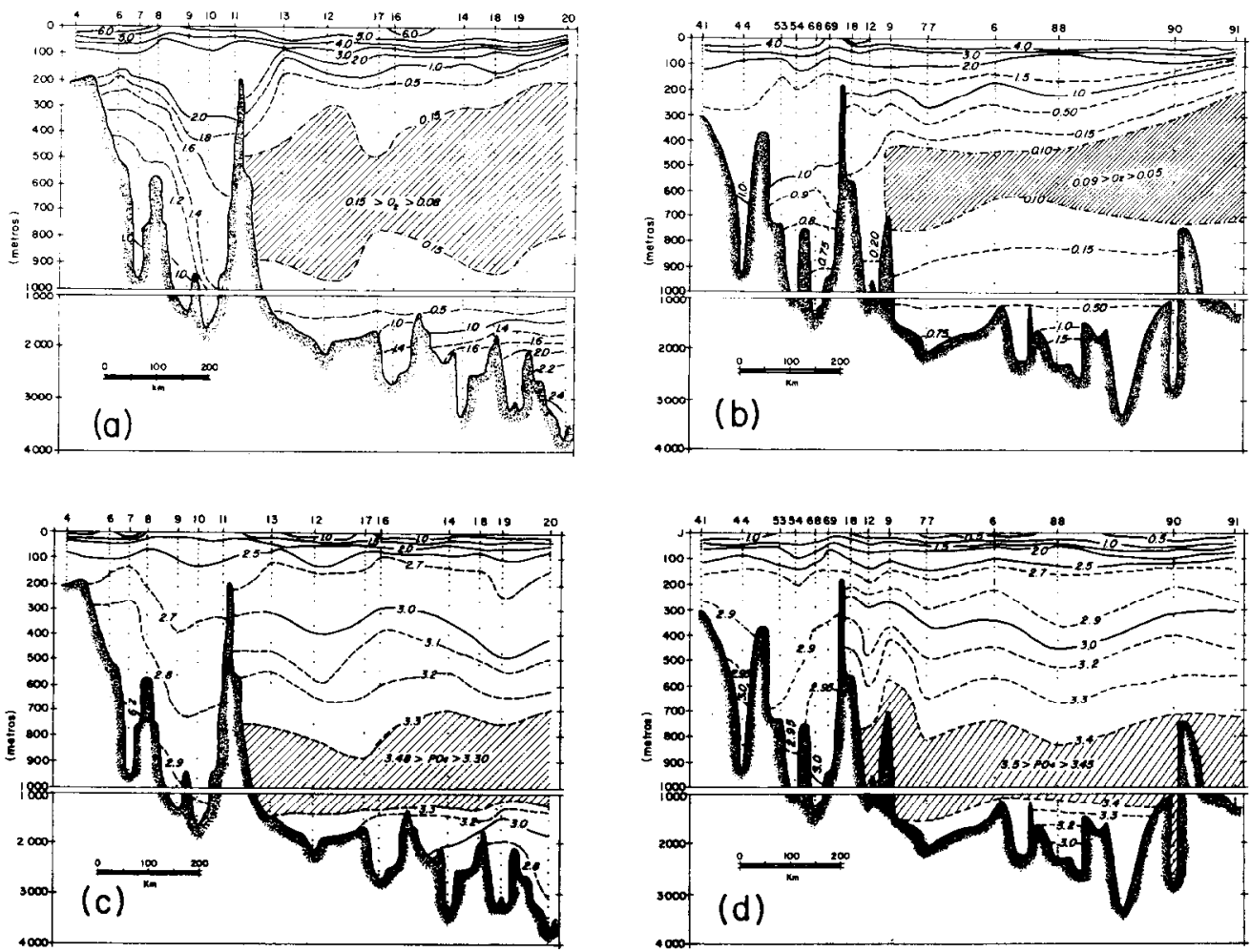

Pig. 3. Distribución vertical de: a) oxígeno disuelto en abrilmayo ( $\mathrm{ml} / \mathrm{l}$ ) (tomada de Gaxiola Castro, Alvarez Borrego y Schwartzlose, 1978); b) oxígeno disuelto en octubre $(\mathrm{ml} / 1)$; fosfato $(\mu \mathrm{M})$ en abril-mayo; y d) fosfato ( $\mathrm{MM}$ ) en octubre. En la parte superior se marcan los números de las estaciones.

En la parte sur del Golfo la termoclina fue más pronunciada en octubre que en abril-mayo (Fig. 2a y b), con temperaturas superficiales entre 6 y $7^{\circ} \mathrm{C}$ mayores en octubre. Las temperaturas fueron mayores en octubre como hasta $300 \mathrm{~m}$. En la parte norte las temperaturas superficiales en octubre fueron 7 a $9^{\circ} \mathrm{C}$ mayores que en abril, pero la diferencia fue sólo $\sim 0.45^{\circ} \mathrm{C}$ a $150 \mathrm{~m}$. En la estación más profunda del canal de Ballenas (est. 68, Fig. 2), las temperaturas fueron mayores en octubre que en abril de la superficie hasta aproximadamente $220 \mathrm{~m}$. Pero de esta profundidad hasta el fondo $(\sim 1500 \mathrm{~m})$ las temperaturas fueron mayores en abril hasta en $0.4^{\circ} \mathrm{C}$. La salinidad se comporta de una manera similar a la temperatura en el canal de Ballenas. La salinidad fue mayor en octubre que en abril de la superficie hasta aproximadamente $150 \mathrm{~m}$ 
(Fig. 2c y d), con diferencia de $0.28 \%$ en la superficte. De $150 \mathrm{~m}$ al fondo de la cuenca las salinidades fueron mayores en abril, hasta en $0.04 \%$. En la parte norte del Golfo las salinidades superficiales fueron mayores en octubre entre $0.15 \mathrm{y} 0.21 \%$, pero a $125 \mathrm{~m}$ los valores de octubre fueron menores, con una diferencia de $0.12 \%$ a $150 \mathrm{~m}$. In la parte sur la salinidad superficial fue mayor en abril que en octubre, con diferencias entre 0.03 y $0.12 \%$. Pero desde $\sim 75 \mathrm{~m}$ a la altura de Guaymas y $\sim 40 \mathrm{~m}$ en la boca del Golfo, hasta aproximadamente $250 \mathrm{~m}$ en ambos casos, hubo mayores salinidades en octubre con diferencias de hasta $0.20 \%$. Por debajo de $250 \mathrm{~m}$ la distribución vertical de salinidad fue prácticamente igual en ambos cruceros, en la parte sur del Golfo. Como una característica notable resalta el mínimo de salinidad a profundidates intermedias $(700-800 \mathrm{~m})$, que es el núcleo del Agua Intermedia del Antártico (Griffiths, 1958; Gaxiola Castro, Alvarez Borrego y Schwartzlose, 1978).

Debido a las mayores temperaturas superficiales y por lo tanto menor solubilidad de los gases, la concentración superficial de oxígeno fue menor en octubre que en abril, en las primeras' decenas de metros (Fig. $3 a \mathrm{y} \mathrm{b}$ ). Sin embargo, en la parte sur la isograma de $1 \mathrm{ml} / 1$ fue consistentemente más profunda en octubre que en abril, con diferencia de $30 \mathrm{~m}$ en la boca $\mathrm{y}$ $120 \mathrm{~m}$ en el centro del Golfo. De 250-300 m hacia abajo la distribución vertical de oxígeno fue prácticamente la misma para ambos cruceros, en la parte sur. En el canal de Ballenas el oxígeno tuvo menor concentración en octubre que en abril en toda la columna de agua, con diferencias de $0.95 \mathrm{ml} / \mathrm{l}$ en la superficie y $\sim 0.45 \mathrm{ml} / \mathrm{I}$ a $1200 \mathrm{~m}$. En la parte somera norte también el oxígeno tuvo menor concentración en octubre en toda la columa de agua con diferencias de $\sim 2.20 \mathrm{ml} / 1$ en la superficie y $\sim 0.90 \mathrm{ml} / \mathrm{l}$ a $150 \mathrm{~m}$.

Las concentraciones superficiales de nutrientes de ambos cruceros se resumen en la tabla 1. Para el fin de nuestra calsificación a "grosso modo" en la tabla I, se considera como la parte sur del Golfo sólo la comprendida entre una línea perpendicular a la costa a la altura de cabo San lucas y otra a la altura de Bahla de La Paz. La parte central está comprendida entre ésta y una IInea que cruze el Golfo entre Guaymas e isla Tiburón. LOs valores superficiales muestran en general la tendencla de aumentar le la boca hacia el norte, con máximos en el canal de Ballenas, disminuyendo en la parte norte. Los valores de abril-mayo fueron en general mayores que

108 de octubre. Esto último no fue muy notable en la parte sur, pero 10 rue en el resto del Golfo.' En octubre 108 gradientes superficiales transversales de nutrientes no muestran efectos de surgencla, es decir, de costa a costa los valores no son significativamente diferentes. 


\begin{tabular}{|c|c|c|c|c|}
\hline & $\begin{array}{l}\text { PARTE } \\
\text { SUR }\end{array}$ & $\begin{array}{l}\text { PARTE } \\
\text { CENTRAL }\end{array}$ & $\begin{array}{l}\text { CANAL DE } \\
\text { BALLENAS }\end{array}$ & $\begin{array}{l}\text { PARTE } \\
\text { NORTE }\end{array}$ \\
\hline \multirow{2}{*}{$\mathrm{PO}_{4} \mu \mathrm{M}$} & $\sim 0.6$ & $\sim 1.0$ & $1.7-2.0$ & $0.8-1.0$ \\
\hline & $\sim 0.4$ & $\sim 0.5$ & $0.9-1.5$ & $0.7-1.0$ \\
\hline \multirow{2}{*}{$\mathrm{NO}_{3} \mu \mathrm{M}$} & $\sim 0.6$ & $\sim 1.9$ & $\sim 13.0$ & $0.2-4.0$ \\
\hline & $\sim 0.1$ & $\sim 0.3$ & $1.0-7.5$ & $0.0-0.2$ \\
\hline \multirow{2}{*}{$\mathrm{NO}_{2} \mu \mathrm{M}$} & 0.0 & $\sim 0.09$ & $\sim 0.31$ & $0.02-0.20$ \\
\hline & $\sim 0.01$ & $\sim 0.01$ & $0.13-0.45$ & $0.00-0.09$ \\
\hline \multirow{2}{*}{$\mathrm{Si} \mathrm{O}_{2} \mu \mathrm{M}$} & $\sim 1.0$ & $0.0-5.0$ & $\sim 29.0$ & $11.0-18.0$ \\
\hline & $\sim 2.4$ & $\sim 2.9$ & $6.6-19.6$ & $6.1-10.2$ \\
\hline
\end{tabular}

Tabla I. Valores superficiales para las diferentes regiones del Golfo $(\mu \mathrm{M})$. Los números superiores corresponden al crucero de abril-mayo, y los inferiores al de octubre.

El nitrito presentó un máximo subsuperficial en la mayoría de las estaciones, con valores de $0.2-0.6 \mu \mathrm{M}$ a $30-80 \mathrm{~m}$. En el canal de Ballenas hubo valores mayores a $1.0 \mu \mathrm{M}$ en este máximo. En algunas estaciones de la boca del Golfo se presentó un segundo máximo de nitrito a $150-400 \mathrm{~m}$, con concentraciones de hasta $0.7 \mu \mathrm{M}$ en abril-mayo y hasta $1.9 \mu \mathrm{M}$ en octubre. Con excepción de estos casos, las concentraciones de nitrito fueron menores a $0.1 \mu \mathrm{M}$ a profundidades mayores de 100 $\mathrm{m}$, con valores bajo el nivel de detectabilidad en muchos casos.

En el centro del norte del Golfo, que es muy somero, en abril se presentaron máximos débiles de fosfato, nitrato $y$ silicato a 80-125 m, con 2.3-2.5 $\mu \mathrm{M}, 21-23 \mu \mathrm{M}$ y 53-67. $\mu \mathrm{M}$ respectivamente. Estos máximos no se detectaron en octubre. En el canal de Ballenas, de acuerdo con la disminución monotónica de oxígeno, los tres nutrientes aumentaron monotónicamente con la profundiad en ambos cruceros, hasta $3.0 \mu \mathrm{M}, 30 \mu \mathrm{M}$ y 70 $\mu \mathrm{M}$ a $1500 \mathrm{~m}$, respectivamente. Aunque las concentraciones de 
nutrientes, a $1500 \mathrm{~m}$ en el canal de Ballenas, fueron consistentemente mayores en octubre que en abril, de acuerdo con los menores valores de oxígeno en octubre, las diferencias no son estadísticamente significativas (v.g.: al $95 \%$ de nivel de confianza) (Pigs. $3 c$ y d y $4 a, b, c y d$ ). A $1000 \mathrm{~m}$, el canal de Ballenas tiene $0.6 \mu \mathrm{M}, 12 \mu \mathrm{M}$ y $60 \mu \mathrm{M}$ menos de fosfato nitrato $y$ silicato respectivamente, que la parte sur del Golfo.

Al sur del umbral sur del canal de Ballenas, la distribución vertical de estos nutrientes es muy similar a la de océano abierto. Fosfato y nitrato tienen un gradiente mu marcado de 0 a $100 \mathrm{~m}$ de acuerdo con el gradiente de oxlgeno. Las isogramas de $2.5 \mu \mathrm{M}$ de fosfato, de $20 \mu \mathrm{M}$ de nitrato $\mathrm{y}$ de $20 \mu \mathrm{M}$ de silicato, se localizaron a mayor profundidad en octubre que en abrilmayo. A $100 \mathrm{~m}$, en abril-mayo, los valores de fosfato fueron entre 2.5 y $2.7 \mu \mathrm{M}, \mathrm{y}$ los de nitrato entre $20 \mathrm{y} 25 \mu \mathrm{M}$; mientras que en octubre los valores de fosfato fueron entre 2.0 y 2.5 $\mu \mathrm{M}, \mathrm{y}$ los de nitrato entre 15 y $22 \mu \mathrm{M}$. Esto no se aprecia claramente en las gráficas aquí presentadas, pero es claro en los datos tabulados. Por debajo de $200-300 \mathrm{~m}$ los tres nutrientes muestran igual distribución vertical para ambos cruceros; algunas diferencias apreciables en las gráficas no son significativas con la precisión de los datos (Figs. 3c y d y $4 a, b, c y d)$. El fosfato tiene un máximo de cerca de 3.5 $\mu \mathrm{M}$ a 800-1200 m, y luego disminuye a cerca de $2.8 \mu \mathrm{M}$ a $3000 \mathrm{~m}$. El nitrato tiene un máximo débil de 43-44 $\mu \mathrm{M}$ a 1000-1300 m, para disminuir luego a cerca de $39 \mu \mathrm{M}$ a 2500-3000 m. El silicato aumenta monotónicamente con la profundidad a cerca de $170 \mu \mathrm{M}$ a $3000 \mathrm{~m}$.

Las distribuciones verticales, transversales al Golfo, de nutrientes y otras propiedades no mostraron en octubre el efecto de surgencias.

\section{DISCUSTONES.}

Mediante la comparación de los datos de ambos cruceros se aprecia una variación estacional en los $300 \mathrm{~m}$ superficiales del Golfo en la parte sur, además de una diferencia apreciable en toda la columna de agua en Cuenca Salsipuedes y al norte de ella. En ambos cruceros las temperaturas superficiales fueron mayores en la boca que en la parte norte del Golfo. De acuerdo con Robinson (1973), de octubre a junio, las temperaturas superficiales en la boca son mayores que en el extremo interno del Golfo. Sin embargo, las salinidades superficiales fueron mayores en el extremo interno en ambos cruceros, debido a la fuerte evaporación y a lo somero de esta parte del Golfo. Entre abril y octubre la termoclina se presenta bien desarroIlada al sur de las islas Angel de la Guarda y Tiburón, con máximo gradiente en agosto (Roden, 1964). En nuestro caso la 

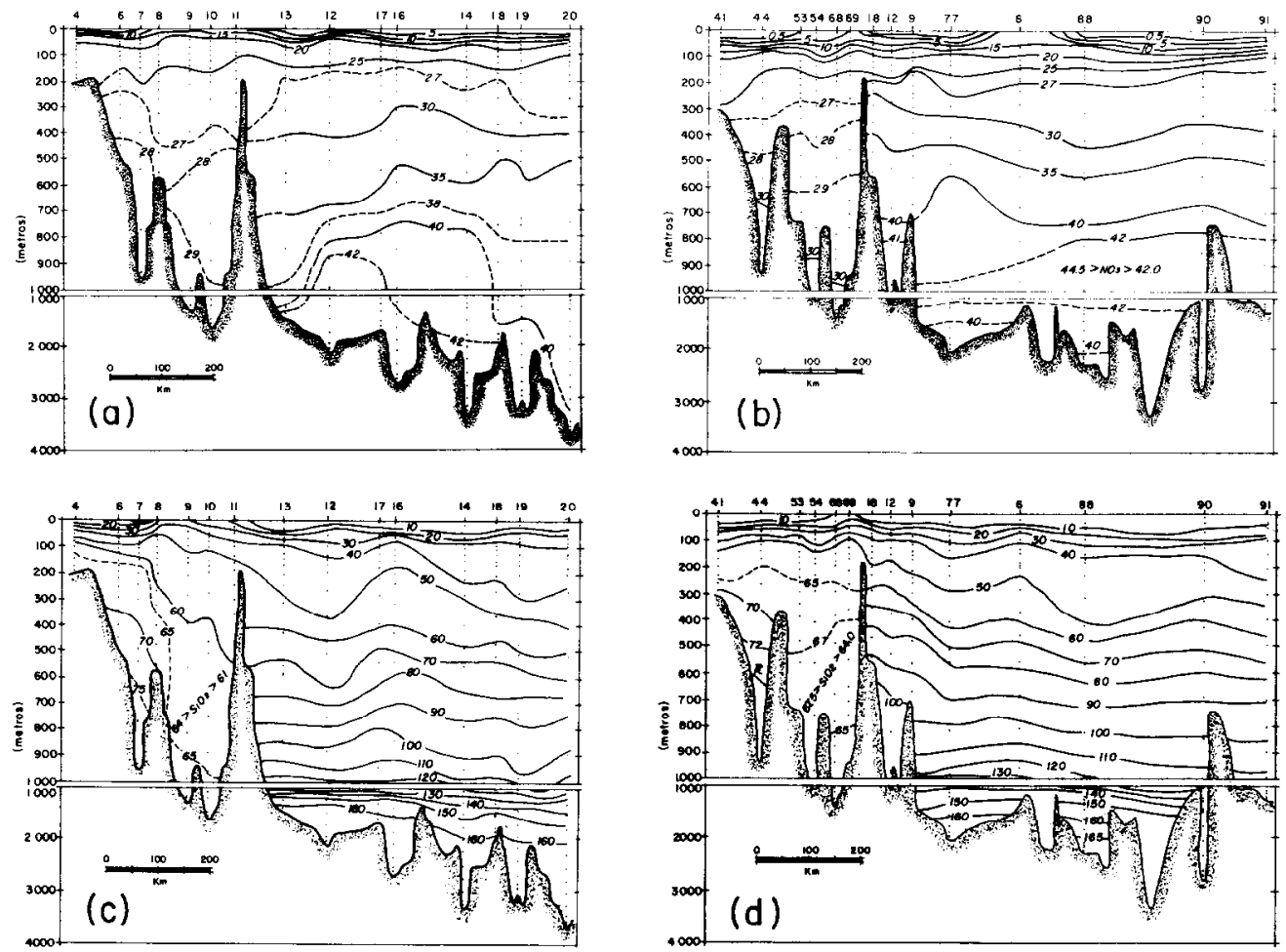

Fig. 4. Distribución vertical de: a) nitrato ( $\mu \mathrm{M})$ en abrilmayo; b) nitrato ( $\mu \mathrm{M})$ en octubre; c) silicato $(\mu \mathrm{M})$ en abril-mayo y d) silicato $(\mu \mathrm{M})$ en octubre. En la parte superior se marcan los números de las estaciones.

termoclina fué más intensa en octubre que en abril. En ambos casos la termoclina fué más intensa en la boca del Golfo que en el interior.

Las salinidades superficiales en la parte sur del Golfo fueron menores en octubre que en abril posiblemente debido a una combinación de los efectos de la precipitación pluvial local de verano y otoño, $y$ de la advección que acarrea agua superficial del sur hacia el Golfo en verano-otoño. La entrada del Golfo de California tiene en la superficie principalmente tres masas de agua: el agua fría de la Corriente de California, de baja salinidad $\left(5^{\circ} / 00 \leq 34.60\right)$, que fluye hacia el sur a 10 largo de la costa oeste de Baja California; el agua caliente del Pacífico Tropical Oriental, de salinidad intermedia (34.65 $\left.\leq S^{\circ} \% 0 \leq 34.85\right)$, que fluye del sureste hacia la boca del Golfo; y el agua caliente y de mayor salinidad ( $\% \%$ ?34.90) del Golfo de California mismo (Roden y Groves, 1959; Griffiths, 1968; Stevenson, 1970). De acuerdo con Granados, Gallegos y Schwartzlose(1974), existe un patrón general de flujo superficial 


\section{NUTRIHPTES EN EE GOLFO DE CATIFORNIA}

hacia el sur en invierno y hacia el norte en verano, en la parte sur del Golfo. El flujo del sur en verano acarrea agua de la zona tropical donde la salinidad ha disminuido por precipitación pluvial en verano y otoño. Esta agua superficial del sur viene además empobrecida en nutrientes por el efecto de fotosíntesis. En la parte sur del Golfo ocurren tormentas violentas entre junio y noviembre provenientes en su mayoría del sur frente al Golfo de Tehuantepec, algunas de las cuales tienen magnitud de huracanes. La ocurrencia de estas tormentas, con duración de unos pocos días cada una, provoca una turbulencia que seguramente afecta la columna de agua en las primeras dos a tres centenas de metros superficiales. Esta debe ser la causa, por lo menos parcialmente, de la mayor profundidad de la isograma de $1 \mathrm{ml} / \mathrm{I}$ de oxígeno en octubre con respecto a la de abril-mayo, y también mayor profundidad de las de $2.5 \mu \mathrm{M}$ de fosfato, $20 \mu \mathrm{M}$ de nitrato y $20 \mu \mathrm{M}$ de silicatos. Por efectos de turbulencia el agua subsuperficial se mezcla con la superficial enriqueciéndose de oxígeno y empobreciéndose de nutrientes. Esto a su vez debe aumentar la concentración de nutrientes en el agua superficial; sin embargo, en abril-mayo la concentración superficial de nutrientes es mayor debido a los procesos de surgencia en invierno y primavera y también al patrón general de flujo del norte al sur que acarrea hacia la boca agua más rica en nutrientes. Con los datos con que se cuenta no se puede estimar cuanto de la diferencia se debe a mezcla y cuanto a adevección. El efecto debe ser combinado además con el de procesos de respiración y fotosíntesis. La mayor salinidad subsuperficial $(70-250 \mathrm{~m})$ en octubre, con respecto a la de abril-mayo, en la parte sur del Golfo, puede deberse a un fenómeno de advección que acarrearía agua del interior del Golfo, con mayor salinidad, hacia la boca del mismo. O bien, puede ser el remanente local del efecto de evaporación de primavera y verano hasta antes del comienzo de las tormentas tropicales. Desgraciadamente la circulación del Golfo es muy compleja y poco conocida todavía para explicar mejor estos fenómenos (Alvarez Borrego, en preparación).

Es interesante notar que debido a lo somero de isogramas como la de $1 \mathrm{ml} / 1$ de oxígeno, $2.5 \mu \mathrm{M}$ de fosfato y $20 \mu \mathrm{M}$ de nitrato, cuya profundidades de 100 a $200 \mathrm{~m}$, las zonas de surgencias del Golfo deben ser las de más alta concentración superficial de nutrientes en el océano. Sobre todo las de surgencias de invierno que ocurren en la costa este del Golfo $\mathrm{y}$ que, de acuerdo con Robinson (1973), se desarrollan mejor que las de verano, que ocurren en la costa oeste.

Las diferencias entre los dos cruceros, de la distribución de las propiedades del agua en la cuenca Salsipuedes, son muy interconsistentes e indican que en octubre había en dicha cuenca una mayor proporción del agua de la profundidad del umbral ( $450 \mathrm{~m}$ ) que en abril. El agua de la cuenca resulta 
de la mezcla del agua de la superficie y la de la profundidad del umbral. El agua de la profundidad del umbral es más pobre en oxígeno, más rica en nutrientes y de menor salinidad y temperatura que el agua superficial. Una mayor proporción de

esta agua en el canal de Ballenas resulta en menor oxígeno, temperatura y salinidad, y mayor concentración de nutrientes en el fondo, en octubre que en abril. Por el sólo efecto de mezcla, en la superficie del canal de Ballenas la concentración de nutrientes debería ser mayor en octubre que en abril. Las concentraciones menores de octubre posiblemente se deben al consumo por fotosíntesis, para lo cual ésta debe ser mayor en verano-otoño que en invierno-primavera.

En la parte norte del Golfo hay procesos mur intensos de mezcla debido a las fuertes corrientes de marea. Las mareas vivas llegan a tener un rango de hasta $10 \mathrm{~m}$ en la cabeza del Golfo (Filloux, 1973). Stocks (1976) calculó la componente $M_{2}$ longitudinal al Golfo, de la corriente de marea, y encontró valores de $\sim 60 \mathrm{~cm} \mathrm{seg}{ }^{-1}$ en la parte norte. Esto concuerda con los valores medidos directamente por Schwartzlose (resultados no publicados). Estos procesos de mezcla y el efecto de advección, que causa circulación en forma de grandes remolinos y algo de flujo de las cuencas Salsipuedes y Tiburón hacia el norte (Granados Gallegos y Schwartzlose, 1974), son responsables de la alta concentración de nutrientes en la parte norte del Golfo. La incidencia de tormentas tropicales en el norte del Golfo es mucho menor que en la parte sur. El. máximo subsuperficial ( $80-125 \mathrm{~m}$ ) de nutrientes detectado en abril, en $1 \mathrm{a}$ parte central del norte del Golfo, posiblemente se deba al efecto combinado de fotosíntesis que consume nutrientes en la superficie $\mathrm{y}$ un movimiento convectivo en invierno que acarrearía agua superficial de la cabeza del Golfo al fondo y hacia el sur. Roden (1964) concluyó que en invierno puede haber hundimiento del agua superficial cercana a la costa hasta $\sim 100 \mathrm{~m}$ en el norte del Golfo. Roden (1964) utilizó una media de temperaturas superficiales mínimas de $13^{\circ} \mathrm{C}$ para sus cálculos, pero Alvarez Borrego, Flores Baez y Galindo Bect (1975) midieron temperaturas menores a $10^{\circ} \mathrm{C}$ en la cabeza del Golfo, con un mínimo de $8.25^{\circ} \mathrm{C}$ en diciembre. Estas temperaturas menores deben producir convección a profundidades mucho mayores a $100 \mathrm{~m}$. Este movimiento convectivo también explicaría las mayores salinidades en abril que en octubre, de $\sim 100 \mathrm{~m}$ al fondo; y además el hecho de que en esta parte del Golfo, en abril la salinidad tiene un mínimo a $-100 \mathrm{~m}$, mientras que en octubre disminuye monotónicamente con la profundidad. La evaporación es alta en todo el año, ya que esta parte del Golfo está rodeada de desiertos. De acuerdo con Roden (1964), Ia precipitación anual en esta zona es menor a $10 \mathrm{~cm}$. 


\section{NUTRIENTES EN EL GOLFO DE CALIFORNIA}

El máximo subsuperficial de nitrito $(30-80 \mathrm{~m})$ es una característica común a todos los océanos y se atribuye a nitrificación bacteriana (Wada y Hattori, 1971), a excreción por fitoplancton (Vaccaro, 1965), o ambas. El segundo máximo de nitrito, a 150$400 \mathrm{~m}$, detectado en la boca del Golfo, es característico de zonas muy deficientes de oxígeno y se debe a denitrificación (Cline y Richards, 1972). Cohen y Gordon (1978) encontraron una correlación negativa entre nitrito y $\mathrm{N}_{2} \mathrm{O}$, abajo de $\mathrm{la}$ termoclina, en el Pacífico Norte Oriental Tropical, y una concordancia entre el máximo secundario de nitrito (a $150-400 \mathrm{~m}$ ) y el mínimo de $\mathrm{N}_{2} \mathrm{O}$, y en base a ello concluyeron que $\mathrm{N}_{2} \mathrm{O}$ se consume por denitrificación. Con concentráciones de oxígeno menores a $0.1 \mathrm{ml} / 1$ se lleva a cabo la denitrificación marina (Goering, 1968). Con los bajos niveles de oxígeno en el sur y centro del Golfo, a 400-500 m, no es explicable por qué sólo en la boca del Golfo se detectó el máximo secundario de nitrito.

Redfield (1934) propuso una relación entre las concentraciones de oxígeno disuelto, bióxido de carbono, nitrato y fosfato en el agua de mar basado en la composición promedio de plancton. Esta relación predice que la razón de oxígeno consumido a producción de nutrientes por oxidación biológica es constante y puede ser representada por la ecuación (Redfield, Ketchum y Richards, 1963):

\section{$\left(\mathrm{CH}_{2} \mathrm{O}\right)_{106}\left(\mathrm{NH}_{3}\right)_{16} \mathrm{H}_{3} \mathrm{PO}_{4}+138 \mathrm{O}_{2} \rightarrow 106 \mathrm{CO}_{2}+122 \mathrm{H}_{2} \mathrm{O}+16 \mathrm{HNO}_{3}+\mathrm{H}_{3} \mathrm{PO}_{4}$}

donde $\left(\mathrm{CH}_{2} \mathrm{O}_{106}\left(\mathrm{NH}_{3}\right)_{16} \mathrm{H}_{3} \mathrm{PO}_{4}\right.$ es una molécula orgánica hipotética promedio, conteniendo carbono, nitrógeno y fósforo en la

proporción en que ocurren en el plancton. Esta ecuación predice que al consumirse 276 átomos de oxígeno disuelto en el agua de mar se producen 106 carbonos, 16 nitrógenos y 1 átomo de fósforo. Alvarez Borrego, Guthrie, Culberson y Park (1975), aplicando análisis de regresión lineal múltiple, encontraron que datos de los oceanos Atlántico y Pacífico son consistentes con el modelo de Redfield. La relación propuesta por Redfield generalmente no puede probarse mediante una gráfica directa entre v.g.: nitrato y fosfato, ya que, además de existir cambios en la concentración de los nutrientes y oxígeno por actividad biológica, diferentes masas de agua pueden tener diferentes valores preformados de estas propiedades (Redfield, 1934 y 1942), 10 cual puede resultar en diagramas no lineales, o con pendiente constante pero diferente a la propuesta por Redfield. Alvarez Borrego (1973) encontró que la relación nitrato: fosfato cambia en el Pacífico Norte con la profundidad, y arriba del mínimo de oxígeno con la latitud y la estación del año. 
La relación nitrato-fosfato en el Golfo de California es casi lineal (Fig. 5). Se aprecia una ligera curvatura con la pendiente aumentando con la profundidad, hasta el máximo de nitrato $y$ fosfato. En la figura 5 casi todos los puntos de valores altos de nutrientes, a la izquierda de la línea recta, corresponden a profundidades mayores a la del máximo de nutrientes. Utilizando el método de los mínimos cuadrados se obtiene una pendiente de $16.2 \pm 0.7$, al $95 \%$ de nivel de confianza, con todos los datos de abril-mayo. Se prefirió construir esta gráfica con los datos de abril-mayo por tener mejor precisión que los de octubre. Este intervalo de confianza es consistente con el valor 16 propuesto por Redfield. En octubre no se presentó una diferencia siginificativa en la relación nitrato-fosfato, con relación a la de abril-mayo. Tanto en abril-mayo como en octubre, la abscisa al origen es 1 LM; es decir, en las aguas superficiales hay concentraciones de fosfato proporcionalmente mucho mayores que los de nitrato. Esto posiblemente indique la presencia en la superficie de compuestos con nitrógeno reducido en concentraciones de aproximadamente 5 a $16 \mu \mathrm{M}$.

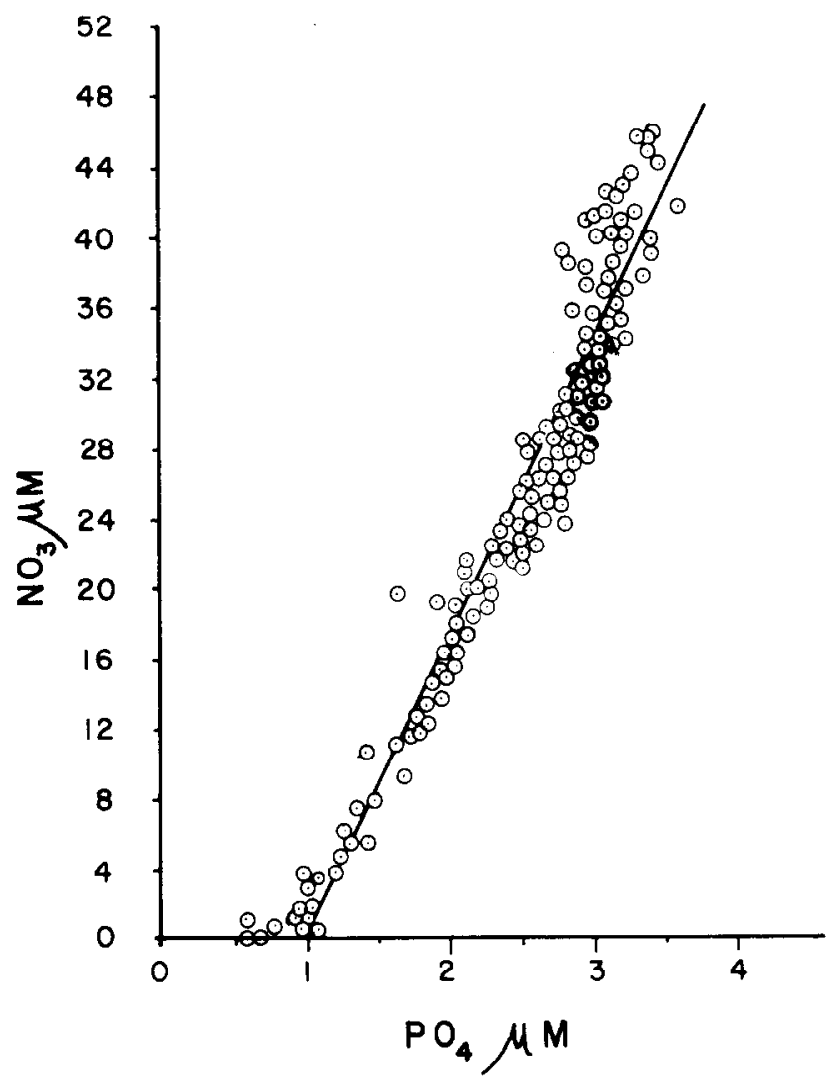

Fig. 5. Diagrama nitrato-fosfato construido con los datos de abril-mayo. 


\section{NUTRIENTHS INT EL GOLFO DE CALIFORNIA}

De Alvarez Eorrego (1973):

$$
\mathrm{NO}_{3}=16 \mathrm{PO}_{4}+\left(\mathrm{P} . \mathrm{NO}_{3}-16 \mathrm{P} \cdot \mathrm{PO}_{4}\right)
$$

Donde $\mathrm{P}_{\mathrm{NO}} \mathrm{N}_{3}$ y $\mathrm{P} . \mathrm{PO}_{4}$ son nitrato y fosfato preformados respetivamente. De la ecuación (1) se deduce que para que haya una relación lineal entre nitrato y fosfato se debe cumplir una de las dos siguientes condiciones: las porciones preformadas son iguales en todas las masas de agua presentes; ó la relación de las porciones preformadas es $\mathrm{P} . \mathrm{NO}_{3}: \mathrm{P} \cdot \mathrm{PO}_{4}=16: 1$. En el Golfo de California la segunda condición es la que se cumple cercanamente, ya que las cantidades preformadas son significativamente diferentes en las diferentes masas de agua presentes (Alvarez Borrego y Schwartzlose, en preparación).

En el Golfo de California se pierden grandes cantidades de silicato de la columna de agua a los sedimentos, a través de la depositación de exoesqueletos de microorganismos, principalmente diatomeas y radiolarios. En ciertas localizaciones, donde el agua en contacto con los sedimentos es muy pobre en oxígeno, se han formado sedimentos laminados que han sido utilizados para estudiar la depositación de silicato (Calvert, 1966). La acumulación de silicato biogénico en las sedimentos de la parte central del Golfo se realiza a una velocidad de ${ }^{m} 10^{13} \mathrm{~g}$ año ${ }^{-1}$. Calvert (1966) indica que el intercambio de agua entre el Golfo y el océano Pacífico provee $\sim 10^{14} \mathrm{~g}$ año ${ }^{-1} \mathrm{de}$ silicato disuelto, y por lo tanto hay suficiente silicato disponible en el agua para suplir el que se acumula en los sedimentos diatomaceos, dado el mecanismo de surgencia que suple silicato a la zona eufótica.

\section{BIBLIOGRAFIA.}

Alvarez Borrego, S. 1973. Oxygen-carbon dioxide-nutrients relationships in the northeastern Pacific Ocean and southeastern Bering Sea. Ph. D. Thesis. School of

Oceanography. Oregon State Univ. Corvallis, Or. 171 p.

Alvarez Borrego, S. Gulf of California. Estuaries and Enclosed Seas. B. H. Ketchum (Editor). Chapter XVI (En preparacion).

Alvarez Borrego, S., B. P. Flores Baez y L. A. Galindo Bect. 1975. Hidrología del Alto Golfo de California II. Condiciones durante invierno, primavera y verano. Ciencias Marinas (Mex.) Vol. 2 (1): 21-36

Alvarez Borrego, S., D. Guthrie, C. H. Culberson and P.K. Park 1975. Test of Redfield's model for the oxygen-nutrient relationships using regression analysis. Limnol. and Oceanogr. Vol. 20: 795-805. 
Alvarez Borrego, S. y R. A. Schwartzlose. Masas de agua del Golfo de California. (En preparación).

Calvert, S. E. 1966. Accumulation of diatomaceous silica in the sediments of the Gulf of California. Geol. Soc. of America Bull. 77: 569-596.

Cline, J. D. and F. A. Richards. 1972. Oxygen deficient conditions and nitrate reduction in the eastern tropical North Pacific Ocean. Limnol. and Oceanogr. 7:885-900.

Cohen, Y. and L. I. Gordon. 1978. Nitrous oxide in the oxygen minimum of the eastern tropical North Pacific: evidence for its consumption during denitrification and possible mechanisms for its production. Deep-Sea Res. Vol. 25: 509524 .

Filloux, J. H. 1973. Tidal patterns and energy balance in the Gulf of California. Nature, 243: 217-221.

Gaxiola Castro, G., S. Alvarez Borrego y R. A. Schwartzlose. 1978. Sistema del bióxido de carbono en el Golfo de California. Ciencias Marinas (Mex), (Esta publicación).

Goering, J. J. 1968. Denitrification in the oxygen minimum layer of the eastern tropical Pacific Ocean. Deep-Sea. Res. 15: 151-164.

Granados Gallegos, J. L. y R. A. Schwartzlose. 1974. Corrientes superficiales en el Golfo de California. Memorias del V Congreso Nacional de Oceanografía. Esc. de Ciencias Marit. del Inst. Tec. de Monterrey. Guaymas, Son.

Griffiths, R. C. 1968. Physical, chemical and biological oceanography of the entrance to the Gulf of California, spring of 1960. U. S. Fish and Wildlife Serv. Sp. Sci. Rp. Fisheries No. 573-47 p.

Redfield, A. C. 1934. On the proportions of organic derivatives in sea water and thelr relation to the composition of plankton. James Johnstone Memorial Volume, íiverpool: 176-192.

Redfield, A. C. 1942. The processes determining the concentration of oxygen, phosphate and other organic derivatives within the depths of the Atlantic Ocean. Papers Phys. Oceanogr. Meteorol. 9 (2): 22 p.

Redfield, A. C., B. H. Ketchum and F. A. Richards, 1963. The influence of organisms on the composition of sea-water. The Sea. M. N. Hill (Editor). Interscience, Vol. 2:26-77. 
Robinson, M. K. 1973. Atlas of monthly mean sea surface and subsurface temperatures in the Gulf of California, Mexico. San Diego Soc. of Nat. Hist. Memoir 5.97 p.

Roden, G. I. 1964. Oceanographic aspects of the Gulf of California. Marine Geology of the Gulf of California: a symposium. van Andel, Ty. H. y G. G. Shor Jr. (editors). Amer. Assoc. Petroleum Geologists. Memoir $3: 30-58$.

Roden, G. I. and G. W. Groves. 1959. Recent oceanographic investigations in the Gulf of California. Jour. Marine. Res. Vol. 18, Num. 1:10-35.

Rusnak, G. A., R. L. Fisher and F. P. Shepard. 1964. Bathymetry and faults of Gulf of California. Marine Geology of the Gulf of California: a symposium. van Andel, Tj. H. and G. G. Shor Jr. (e⿳亠丷itors). Amer. Assoc. Petroleum Geologists. Memoir 3: 59-75.

Scripps Institution of Oceanography. 1974. Marine Technician's Handbook. Nutrient analysis. Inst. Mar. Res. SIO Ref. No. 71-8. Sea Grant. Pub. No. 9:1-29.

Stevenson, M. R. 1970. On the physical and biological oceanography near the entrance to the Gulf of California, October 1966-August 1967. Inter-Amer. Trop. Tuna Comm. Bull. 4, Num. 3:389-504.

Stocks, G. G. 1976. Modeling of tides and tidal dissipation in the Gulf of California. Ph. D. Thesis. Scripps Inst. of Ocean. Univ. of Calif. San Diego. La Jolla, Calif. $102 \mathrm{p}$.

Sverdrup, H. U. 1941. The Gulf of California; preliminary dicussion on the cruise of the E. W. Scripps in Februay and March 1939. 6th. Pacific Sci. Cong. Proc. Vol. 3: $161-166$.

Thomas, W. H. and A. N. Dodson. 1968. Effects of phosphate concentration on cell division rates and yield of a tropical oceanic diatom. Biol. Bull. Mar. Biol. Lab. Woods Hole, 134: 199-208.

Vaccaro, R. R. 1965. Inorganic nitrogen in sea water. Chemical Oceanography. J. P. Riley and G. Skirrow (Editors). Acad. P. la. ed. vol. 1: 365-404. 
ALVAREZ BORREGO - RIVERA - GAXIOLA CASTRO - ACOSTA RUIZ - SCHWARTZLOSE

Wada, E., and A. Hattori. 1971. Nitrite metabolism in the euphotic layer of the central North Pacific Ocean. Limnol. and Oceanogr. 16: 766-772.

Recibido: diciembre 22 de 1978. 